\title{
A New Energy-Efficient Data Transmission Scheme Based on DSC and Virtual MIMO for Wireless Sensor Network
}

\author{
$\mathrm{Na} \mathrm{Li},{ }^{1}$ Liwen Zhang, ${ }^{1}$ and Bing $\mathrm{Li}^{2}$ \\ ${ }^{1}$ School of Electric Engineering, Henan University of Science and Technology, Luoyang 471003, China \\ ${ }^{2}$ School of Information Engineering, Henan University of Science and Technology, Luoyang 471003, China
}

Correspondence should be addressed to Bing Li; lbking89@163.com

Received 30 September 2014; Accepted 5 February 2015

Academic Editor: Kalyana C. Veluvolu

Copyright (C) $2015 \mathrm{Na} \mathrm{Li}$ et al. This is an open access article distributed under the Creative Commons Attribution License, which permits unrestricted use, distribution, and reproduction in any medium, provided the original work is properly cited.

Energy efficiency in wireless sensor network (WSN) is one of the primary performance parameters. For improving the energy efficiency of WSN, we introduce distributed source coding (DSC) and virtual multiple-input multiple-output (MIMO) into wireless sensor network and then propose a new data transmission scheme called DSC-MIMO. DSC-MIMO compresses the source data using distributed source coding before transmitting, which is different from the existing communication schemes. Data compression can reduce the length of the data and improve the energy efficiency. In addition, DSC-MIMO does not require the cluster heads to collect the data of the source nodes, which reduces the frequencies of data transmission and saves energy. In the simulation, we analyze the energy consumption of DSC-MIMO. The results indicate that DSC-MIMO can effectively reduce the energy consumption and improve the energy efficiency of the whole wireless sensor network.

\section{Introduction}

In recent years, wireless sensor network (WSN) has achieved great progress with more and more new technology being applied to it, which improves the suitability and diversity of WSN application. However, the limiting energy of WSN nodes is still not solved and energy saving is still a major problem. Energy consumption in WSN is mainly caused by the communication of the nodes; thus reducing the communication node is the primary means for energy saving. Particularly, in large-scale WSN, communication energy consumption increases dramatically with the increasing communication distance. If a certain number of nodes prematurely deplete their energy, they will segment the network into a lot of islands, which can seriously affect the reliability of wireless sensor networks. In order to enhance the reliability of WSN, it is necessary to increase the density of nodes. However, the correlation of data collected by adjacent nodes also increases with the increasing density of nodes. These high relevant data are transferred directly to the data gather node (DGN), which seriously reduces the energy efficiency of WSN.

Numerous studies indicate that virtual MIMO technology can effectively save energy consumption of nodes communication and extend the communication range [1]. The researchers have made a lot of excellent virtual MIMO communication scheme. Cui et al. proposed a model of virtual MIMO communication in single-hop wireless sensor networks with Alamouti coding [2]. They analyzed the energy consumption of the network and the data propagation delay and then compared the energy consumption of virtual MIMO with SISO's in the same bit error rate requirement. Their research indicated that the virtual MIMO was more suitable than SISO in the long-distance data transmission according to energy efficiency and data transfer delay. Jayaweera analyzed the effect of the modulation constellation size, the transmission distance, the increasing control overhead of the training sequence, and delay performance of virtual MIMO in wireless sensor networks [3]. The research further proved that the virtual MIMO communication was more energy efficient than SISO communication and significantly reduced the transmission delay with selecting the appropriate parameters. V-BLAST space-time coding was introduced into the virtual MIMO communication by Jayaweera [4], which could avoid the cooperating encoding process of the transmitting nodes and further improve energy efficiency. Bravos and Kanatas analyzed and compared the energy consumption of 
virtual MIMO and multihop SISO [5]. The results showed that energy efficiency of the two methods depends on the density of network nodes, wireless channel condition, and the distance to the sink node. The virtual MIMO was better than multihop SISO in certain condition. Rafique et al. combined virtual MIMO and multicarrier modulation and analyzed the effect of different virtual MIMO communication modulation schemes. The research verified that the BPSK-WOFDM was a very useful and energy-efficient modulation scheme for high-speed virtual MIMO transmission [6]. Krunz et al. proposed a novel virtual MIMO communication strategy, called CMIMO (cooperative MIMO) [7], which involved clustering the WSN into several clusters, and each cluster was managed by two cluster heads ( $\mathrm{CHs}$ ): a master $\mathrm{CH}$ $(\mathrm{MCH})$ and a slave $\mathrm{CH}(\mathrm{SCH})$. CMIMO achieves energyefficient multihop transmission, by proper selection of the $\mathrm{MCH}$ and SCHs, adaptation of the antenna elements and power in the intercluster communications phase, and using a cross-layer MIMO-aware route selection algorithm. The experimental results showed that CMIMO could significantly reduce energy consumption and extend the network lifetime. Nasim et al. proposed an energy-efficient hierarchical cooperative clustering scheme for wireless sensor networks [8]. According to this protocol, nodes cooperated to form clusters at each level of network hierarchy ensuring maximal coverage and minimal energy expenditure with relatively uniform distribution of load within the network. Simulation results indicated that this protocol could effectively reduce the number of hops in multihop transmission and achieve energy-saving purpose.

These virtual MIMO communication schemes can save the communication energy consumption, but they cannot solve energy efficiency problem in high-density WSN caused by high correlation source data. Aiming at this problem, we introduce distributed source coding (DSC), which can compress the high correlation source data before sending, into the virtual MIMO-based WSN and then propose a new communication scheme, called DSC-MIMO, which can improve energy efficiency and reduce communication energy consumption significantly. DSC-MIMO is based on cooperative group (CG) and DSC. Each CG, composed of two correlative source nodes, compresses the source data using DSC firstly and then sends the compressed data directly through virtual MIMO link to the next hop receiver without forwarding data to the cluster head $(\mathrm{CH})$, which can reduce forwarding times of source data and improve the energy efficiency.

DSC is based on the theorem proved by Slepian and Wolf in the 1970s [9]. The fascinating aspect of DSC is that efficient compression of two or more sources can be achieved by separate encoding and joint decoding. Slepian and Wolf give lossless compression theoretical limit of DSC for two related sources using information entropy, called S-W limit. For example, there are two related sources, $X_{1}$ and $X_{2}$, which are separately encoded according to Slepian and Wolf theories with the code rates being, respectively, $R_{X 1}$ and $R_{X 2}$. The region of $R_{X 1}$ and $R_{X 2}$, as shown in Figure 1, is bounded by the following inequalities:

$$
R_{X 1} \geq H\left(X_{1} \mid X_{2}\right),
$$

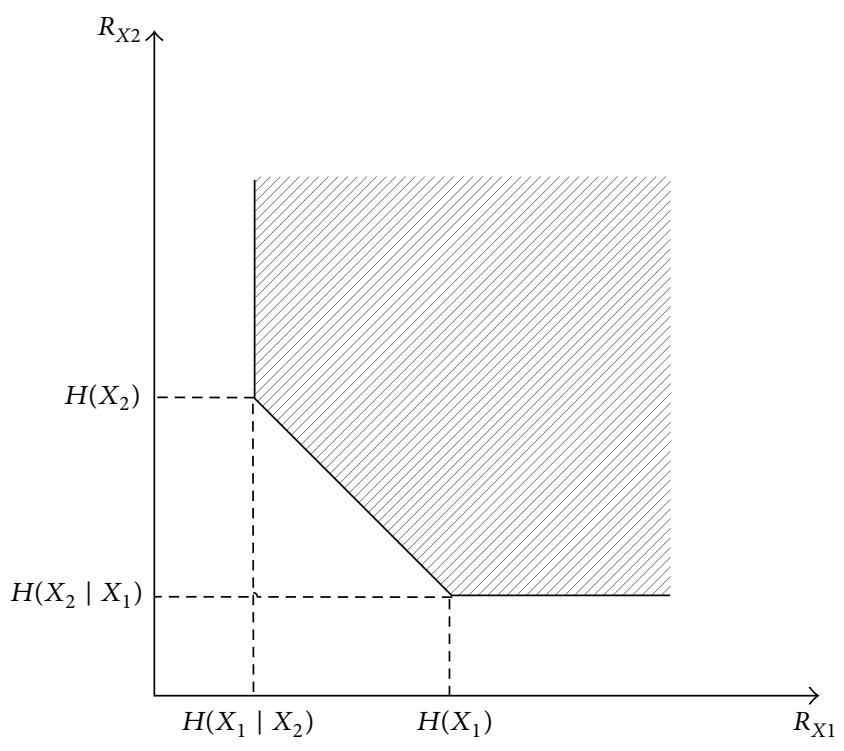

FIGURE 1: Rate region for Slepian-Wolf coding (two sources).

$$
\begin{aligned}
& R_{X 2} \geq H\left(X_{2} \mid X_{1}\right), \\
& R_{X 1}+R_{X 2} \geq H\left(X_{1}, X_{2}\right) .
\end{aligned}
$$

Due to the good performance in low coding complexity, good rate-distortion, and error resilience, DSC has been the widespread concern of scholars. There have also been a lot of excellent DSC schemes, which can be roughly divided into two categories: asymmetric DSC and symmetric DSC.

For asymmetric DSC scheme, the most representative is distributed source coding using syndromes (DISCUS) [10]. In DISCUS, one source transmits its syndromes to achieve data compression; another source sends its raw data directly as side information. At the receiving end, the decoder recovers the compressed data through joint decoding, using the received the side information and the syndromes. On the basis of DISCUS, researchers have proposed a number of asymmetric DSC schemes using turbo codes and low-density parity check (LDPC) [11-14].

For asymmetric DSC scheme, the most representative is the scheme proposed by Sartipi [15]. In Sartipi’s scheme, two sources are encoded using LDPC firstly. Then one source sends its corresponding parity bits and only the first half of the information bits, and the other sends its corresponding parity bits and the second half of the information bits. The decoder recombines the two parts of information bits and then uses message passing decoding algorithm to recover the original data. Later, Sartipi extends this scheme to achieve a different code rate [16].

\section{Network Model}

Before describing how DSC-MIMO works, we set up a wireless sensor network with the following features.

(i) The study object of this paper is WSN with high node density and large scale, which uses clustering 


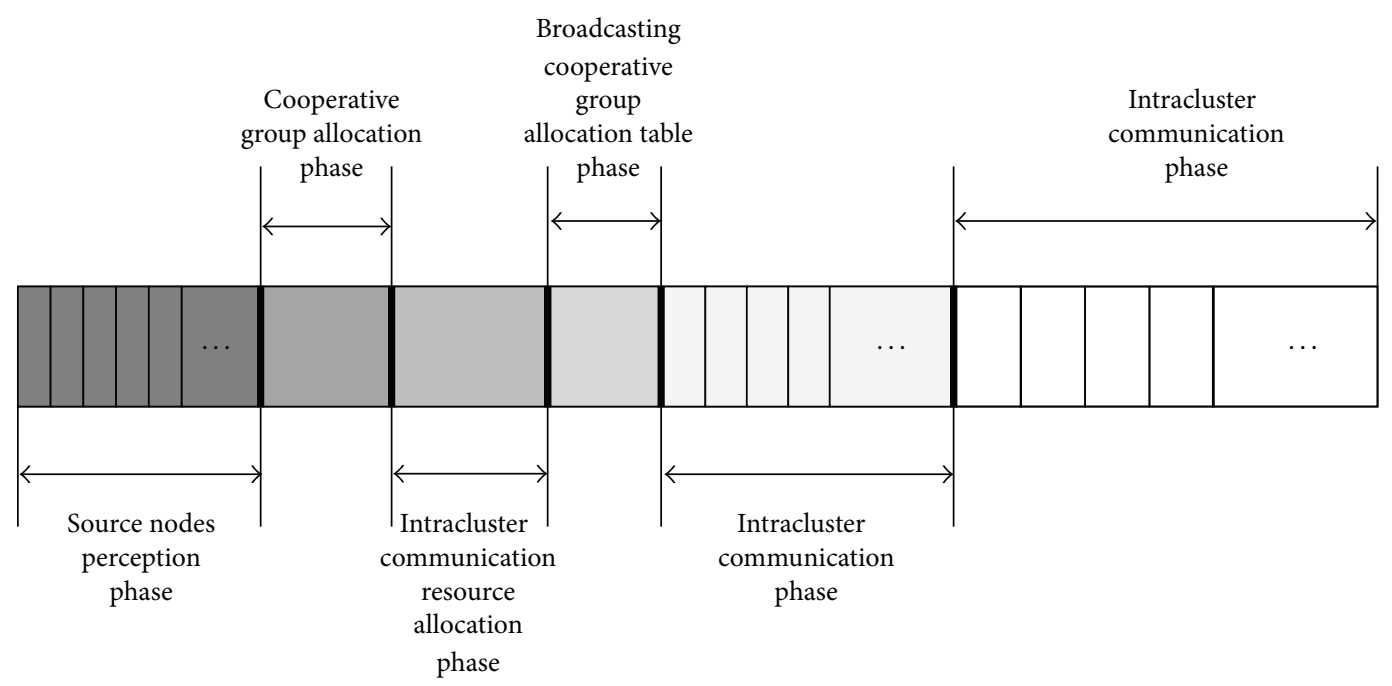

Figure 2: A communication cycle of DSC-MIMO.

approach to management and data collected by nodes of the same cluster are high correlation so that they can be compressed through DSC.

(ii) The data collected by network nodes are finally gathered together at the data gather node (DGN), which is a dual-antenna sending and receiving end with enough energy and a powerful processor.

(iii) All of the network nodes are evenly distributed in the assigned area in which any two neighbor nodes can form a virtual dual-antenna system and the wireless channel between the nodes is symmetric.

(iv) The maximum transmission power of each node is defined as $P_{\max }$. During communication, the nodes can adjust their communication distance by adjusting the transmission power.

\section{The DSC-MIMO}

DSC-MIMO clusters the wireless sensor network into several clusters and its clustering process is similar to CMIMO [7]. In the clustering process, the master cluster heads (MCHs) are generated through election and then, respectively, choose vice cluster heads (VCHs) from their neighbors. In DSCMIMO, MCHs divide the source nodes into several cooperative groups, and each group, consisting of two adjacent source nodes, plays the role of a virtual dual-antenna transmitter, which can transmit the source data directly; $\mathrm{VCH}$ and $\mathrm{MCH}$ are always seen as a cooperative group, called master cooperative group (MCG), which can receive and forward the data from CGs of other clusters. MCGs are the foundation of multihop virtual MIMO transmission. After the clustering is completed, DSC-MIMO starts to build the routing table for the entire network. The routing table building algorithm of DSC-MIMO, similar to CMIMO, is based on Dijkstra algorithm, which can build the shortest routing path for each cluster. Then, DSC-MIMO officially enters periodic communication process. A communication cycle is divided into six phases: source nodes perception phase, cooperative groups' allocation phase, intercluster communication resource allocation phase, broadcasting cooperative group allocation table phase, intracluster communication phase, and intercluster communication phase, which is shown in Figure 2.

In source nodes perception phase, the source nodes of a cluster send "Data Coming" message to the $\mathrm{MCH}$ at different time slots through a competitive manner, telling the $\mathrm{MCH}$ their own IDs, locations, residual energy, and so on. If $\mathrm{MCH}$ or $\mathrm{VCH}$ is also the source node, it will participate in the coming cooperative groups allocation phase and constitute CGs with other general source nodes. However, it does not undermine the MCG which always exists. When MCG has to forward data from CGs of other clusters, it will be allocated the appropriate communication resources to finish the same communication process like the general CGs.

In cooperative group allocation phase, $\mathrm{MCH}$ divides source nodes into several groups according to the cooperative group allocation algorithm proposed in this paper and then allots time slots for intracluster communication to each $\mathrm{CG}$. When the allocation process completes, $\mathrm{MCH}$ generates a cooperative group allocation table which contains the group information and time slots allocation information for intracluster communication. Due to the randomness of locations and number of the source nodes in the cluster, there may be some source nodes which could not be assigned to any cooperative groups. If this happens, the source nodes without CGs will abandon this communication cycle and wait the new cycle coming. If $\mathrm{MCH}$ does not generate cooperative group allocation table, all the nodes in addition to the MCG of this cluster will give up this communication cycle and go into sleeping state.

In intercluster communication resource allocation phase, the MCHs, having the cooperative group allocation table, start to apply intercluster communication slots for each CG according to routing table established before. Every $\mathrm{MCH}$, using CSMA/CA, sends "Channel Request" message to 
the next hop $\mathrm{MCH}$ to request time slots for intercluster communication. "Channel Request" message contains the number of CGs and the occupied time slots. The MCHs, receiving the "Channel Request" message, allocate the intercluster communication resource on the basis of minimum energy consumption and reply with "Channel Response" message. "Channel Response" message contains the information of the assigned time slots. After the data exchange, each $\mathrm{MCH}$ establishes an intercluster communication resource allocation table for its own CGs. If a CG is not assigned time slot, the $\mathrm{MCH}$ will remove it from the cooperative group table. After that, $\mathrm{MCH}$ updates cooperative group allocation table, based on the intercluster communication resource allocation table and the routing table. Then cooperative group allocation table contains group information, intracluster communication resource allocation table, intercluster communication resource allocation table, and the routing information for next hop.

In broadcasting cooperative group allocation table phase, every $\mathrm{MCH}$ broadcasts the table to its source nodes. According to information on the table, all the nodes, assigned cooperative groups, enter into the intracluster communication phase; others give up this communication cycle and go into sleeping status until the next cycle coming.

In intracluster communication phase, the source nodes form cooperative groups based on cooperative group allocation table and finish the DSC using the scheme proposed in [15]. For example, the two nodes in a CG are, respectively, $X$ and $Y$ that both have $k$ bits source data to transmit. The encoding process of $X$ is as follows: $X$ is fed into a systematic LDPC encoder with a rate $R_{1}$. At the output of the encoder, it sends the corresponding parity bits $P_{X}$ and only the first half of the information bits. This results in source encoding rate of $R_{X}=\left(k / 2+P_{X}\right) / k$, which imposes the rate of the systematic LDPC code to be equal to $R_{1}=k /\left(k+P_{X}\right)=1 /\left(R_{X}+1 / 2\right)$. The compression process of the source node $Y$ is similar to that of $X$, with the modification that for the second source we send the second half of the information bits along with the corresponding parity bits. Since the compression rates of both sources are the same, the rates of the corresponding systematic LDPC codes are identical. Therefore, we only need to design a single LDPC code for compressing two correlated sources at the symmetric rate with DSC. After finishing the DSC, the two source nodes in each CG exchange the compressed data adopting RTS/CTS/ACK approach in the assigned time slot and complete space-time coding. During data exchange, the source nodes can adjust their transmit power according to the distance information in the table. On the one hand power adjustment can reduce the data collision, and on the other hand it can save energy. CGs, completing the space-time coding, go into sleeping state until the intercluster communication phase coming. The intracluster communication process of a CG is shown in Figure 3.

In intercluster communication phase, every CG establishes virtual MIMO link with the next hop MCG and completes source data transmission when the assigned time slot comes. MCG, receiving the source data, forwards it to its next hop MCG and realizes the multihop virtual MIMO transmission in the new communication cycle. Figure 4 shows

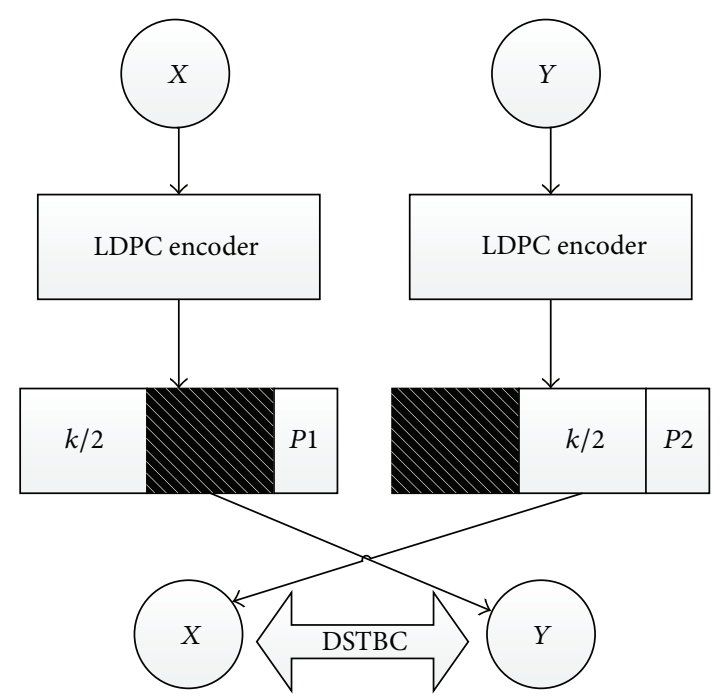

FIGURE 3: The intracluster communication process of a CG.

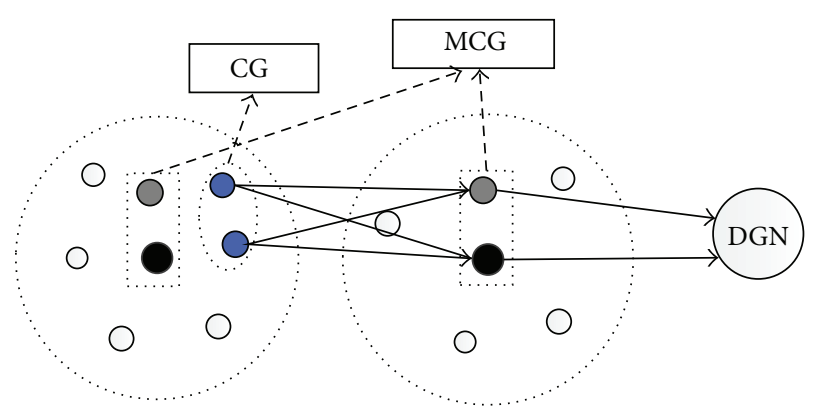

FIgUre 4: A two-hop virtual MIMO transmission of DSC-MIMO.

a multihop virtual MIMO transmission link to DGN in DSCMIMO. CGs, completing the intercluster communication, are dissolved and the nodes go into sleeping state until the arrival of new cycle. When the MCGs received the compressed data from the ordinary CGs, they do not decompress the data and forward it to the next hop MCG directly. The compressed data will be decoded at the DGN using the same decoding method in [15].

From the analysis above, DSC-MIMO has an important feature that source nodes in a CG exchange the data with each other and compete the space-time coding in intracluster communication phase. The source data are transmitted once. However, the source data are transmitted twice for completing the space-time coding in most existing virtual MIMO communication strategies. Therefore, DSC-MIMO can reduce the energy consumption of intracluster communication and improve the energy efficiency. DSC-MIMO makes the CGs and their next hop MCGs form virtual MIMO links for intercluster communication, which is similar to CMIMO. In addition, the two related source nodes in a CG can use the simple and efficient DSC scheme proposed in [15] directly, which can compress the source data and improve the energy efficiency. 
CG is the basic communication unit for DSC-MIMO. How to allocate cooperative groups is an important problem. Therefore, cooperative group allocation algorithm will be discussed in detail below.

\section{Cooperative Group Allocation Algorithm}

CG is the most basic communication unit in DSC-MIMO, so how to divide CGs has a great impact on the performance of communication in WSN. The cooperative croup allocation algorithm proposed in this paper divides the source nodes according to two conditions: communication range and communication quality.

4.1. Communication Range. In order to ensure the coverage of the WSN, the nodes need to have a large range of data transmission. However, energy consumption will increase so quickly that the nodes will die early because of their limited energy if the communication range is excessive. In order to balance energy consumption and communication range, we set a maximum transmission power $P_{\max }$ for every node. Correspondingly, the maximum communication range of a node is limited to $d_{\max }$.

As the two nodes constructing a CG need to exchange data in intracluster communication phase to complete spacetime coding, the distance between the two nodes cannot exceed the maximum communication range $d_{\max }$. Then, we get first qualification for cooperative group allocation algorithm:

$$
d \leq d_{\max }
$$

where $d$ is the distance between two nodes consisting a CG; $d_{\max }$ is the maximum communication range of the nodes, which can be changed by setting the maximum transmission power $P_{\max }$.

4.2. Communication Quality. With the limitation in energy, computing, volume, and so forth, it is very difficult for nodes to know the exact channel state information during communication, especially intercluster communication. Therefore, the cooperative group allocation algorithm generates the CGs with the purpose of ensuring the quality of the virtual MIMO communication in the condition of not knowing channel state information.

A CG is a virtual dual-antenna transmitter, and its transmission data are $\mathbf{x}=\left[\mathbf{x}_{1}, \mathbf{x}_{2}\right]$; the receiving MCG is a virtual dual-antenna receiver, and its receiving data are $\mathbf{y}=$ $\left[\mathbf{y}_{1}, \mathbf{y}_{2}\right]$. The relationship between $\mathbf{x}$ and $\mathbf{y}$ can be expressed as

$$
\mathbf{y}=\mathbf{H x}+\mathbf{n},
$$

where $\mathbf{H}$ is a $2 \times 2$ matrix, indicating virtual MIMO communication channel gains; $\mathbf{n}$ is a vector, representing the noise in the channel. The larger the $\mathbf{H}$ is, the better the virtual MIMO communication quality is. Therefore, the second qualification of cooperative group allocation algorithm is as follows:

$$
\max \{\mathbf{H}\} \text {. }
$$

However, $\mathbf{H}$ is not known when the channel state information is unknown. Therefore, (4) cannot be directly applied. According to the cooperative node selection algorithm in [17], it is effective that selecting the farthest neighbor node as cluster head's cooperative node to ensure the quality of the virtual MIMO communication when the channel state information is unknown. Therefore, we can get the equivalent form of (4):

$$
\max \{\mathbf{H}\} \Longleftrightarrow \max \{d\},
$$

where $d$ is the distance between two nodes consisting a CG.

4.3. The Process of Cooperative Group Allocation. According to the above two conditions, $\mathrm{MCH}$ splits all sources' nodes into groups. First, MCH randomly selects a source node $i$. Then, $\mathrm{MCH}$ selects another source node $j$, whose distance to node $i$ meets the conditions (1) and (4), to form a collaborative group with node $i$. If MCH cannot find the node $j$, node $i$ will give up this collaborative group allocation phase. When $\mathrm{MCH}$ checks all of the source nodes, it can form a collaborative group allocation table. The execution of cooperative group allocation algorithm is shown in Algorithm 1.

\section{Energy Consumption of DSC-MIMO}

In order to analyze energy consumption of DSC-MIMO, we must establish energy consumption model of wireless sensor network node firstly. In data transmission, the total power consumption can be divided into two main components [2, $3,7]$ : the power consumption of all the power amplifiers $P_{\mathrm{PA}}$ and the power consumption of all other circuit blocks $P_{C}$.

$P_{\mathrm{PA}}$ can be expressed as

$$
P_{\mathrm{PA}}(d)=(1+\alpha) P_{\mathrm{out}}(d),
$$

where $d$ is the distance of nodes; $\alpha$ is a factor related to the drain efficiency; $P_{\text {out }}(d)$ can be calculated using the following formula:

$$
P_{\text {out }}(d)=\bar{E}_{b} R_{b} \frac{(4 \pi)^{2} d^{k} M_{l} N_{f}}{G_{t} G_{r} \lambda^{2}},
$$

where $k$ is the path loss; $G_{t}$ and $G_{r}$ are the antenna gains; $\lambda$ is the wavelength; $M_{l}$ is the link margin for compensating the hardware process variations and the other additive background noise or interference; $N_{f}$ is the receiver noise figure; $R_{b}$ is the bit rate; $\bar{E}_{b}$ is the average energy per bit required for a given bit-error rate (BER), which can be calculated using the following equation:

$$
\begin{aligned}
\bar{P}_{b}= & \frac{1}{2^{N_{T} N_{R}}}\left(1-\frac{1}{\sqrt{1+2 N_{0} / \bar{E}_{b}}}\right)^{N_{T} N_{R}} \\
& \times \sum_{k=0}^{N_{T} N_{R}-1} \frac{1}{2^{k}}\left(\begin{array}{c}
N_{T} N_{R}-1+k \\
k
\end{array}\right)\left(1+\frac{1}{\sqrt{1+2 N_{0} / \bar{E}_{b}}}\right)^{k},
\end{aligned}
$$




\section{(1) for all $i \in H$ do $\quad / * i$ represents a source node; \\ $H$ represents all the source node without traversal $*$ I}

(2) mark $i$ as traversal, and update $H$;

(3) for all $j \in H$ do $\quad / * j$ represents a source node $* /$

(4) calculate the distance $d$ between $i$ and $j$;

(5) end for

(6) select the node $k$ whose $d$ satisfies Condition 1 and Condition 2; /* Condition 1: $d<d_{\max }$;

(7) if $k$ not unique then

Condition 2: $\max \{d\}$ 。

(8) randomly select a node from $k$ and compose a CG with $i$;

(9) store this CG in the allocation table;

(10) mark this node as traversal, and update $H$;

(11) else if $k$ unique then

(12) $k$ and $i$ compose a CG;

(13) store this CG in the allocation table;

(14) $\operatorname{mark} k$ as traversal, and update $H$;

(15) else if $k$ none then

(16) end this cycle;

(17) end if

(18) end for

Algorithm 1: Cooperative group allocation algorithm. where $\bar{P}_{b}$ is the given BER; $N_{T}$ and $N_{R}$ are the numbers of the sending node and the receiving node; $N_{0}$ is the noise power density.

$P_{C}$ can be expressed as

$$
\begin{aligned}
P_{\mathrm{C}} \approx & N_{T}\left(P_{\mathrm{DAC}}+P_{\mathrm{mix}}+P_{\text {filt }}\right)+2 P_{\text {synth }} \\
& +N_{R}\left(P_{\mathrm{LNA}}+P_{\mathrm{mix}}+P_{\mathrm{IFA}}+P_{\mathrm{filr}}+P_{\mathrm{ADC}}\right),
\end{aligned}
$$

where $P_{\mathrm{DAC}}, P_{\mathrm{mix}}, P_{\mathrm{LNA}}, P_{\mathrm{IFA}}, P_{\text {filt }}, P_{\text {filr }}, P_{\mathrm{ADC}}$, and $P_{\text {synth }}$ are the power consumption values for the digital-to-analog converter (DAC), the mixer, the low-noise amplifier (LNA), the intermediate frequency amplifier (IFA), the active filters at the transmitter side, the active filters at the receiver side, the analog-to-digital converter (ADC), and the frequency synthesizer, respectively.

Finally, the total energy consumption per bit can be expressed as

$$
E_{\mathrm{pb}}(d)=\frac{P_{\mathrm{PA}}(d)+P_{\mathrm{C}}}{R_{b}} .
$$

The energy consumption of DSC-MIMO focuses on the transmission of source data, which happens in intracluster communication phase and intercluster communication phase. Next, we use (9) to analyze the energy consumption of the two phases.

5.1. Intracluster Communication Phase. According to the previous description, the nodes, belonging to a CG, exchange their data after DSC using SISO mode. Therefore, the energy consumption for transmitting 1-bit original source data is

$$
E_{\mathrm{pb} \_\mathrm{DSC}}^{\mathrm{SISO}}(d)=\left.R_{\mathrm{DSC}} E_{\mathrm{pb}}(d)\right|_{N_{T}=N_{R}=1},
$$

where $R_{\mathrm{DSC}}$ is the compressing rate of DSC; we can set it the same as [15], 75\%.

5.2. Intercluster Communication Phase. In the intercluster communication phase, every CG communicates with the next hop MCG through a $2 \times 2$ virtual MIMO link. Therefore, the energy consumption for transmitting 1-bit original source data is

$$
E_{\mathrm{pb}}^{\mathrm{MIMO}}(d)=\frac{R_{b}^{\mathrm{eff}}}{R_{b}}\left[\left.E_{\mathrm{pb}}(d)\right|_{N_{T}=2, N_{R}=2}\right] R_{\mathrm{DSC}},
$$

where $R_{b}^{\text {eff }}$ is the effective bit rate, which is expressed as

$$
R_{b}^{\mathrm{eff}}=\frac{\left(F-p N_{T}\right)}{F} R_{b}
$$

where $F$ is the block size of space-time coding and $p$ is the training overhead factor.

\section{Simulation Analyses}

To verify the energy conservation effect of DSC-MIMO, we establish a simulation platform to compare the energy consumption with CMIMO in different communication phases. The main simulation parameters are shown in Table 1, which are the same as [7]. In addition, we set the rate of DSC as 75\% which is the same as [15].

Figure 5 shows the communication energy consumption of DSC-MIMO and CMIMO in the intracluster communication phase. To be fair, the control overhead of DSCMIMO is counted to the energy consumption of intracluster communication during the simulation. The result shows 
TABLE 1: Simulation parameters.

\begin{tabular}{lc}
\hline$\lambda=0.125 \mathrm{~m}$ & $\alpha=0.47$ \\
$G_{t} G_{r}=5 \mathrm{dBi}$ & $N_{0}=-174 \mathrm{dBm} / \mathrm{Hz}$ \\
$B=10 \mathrm{KHz}$ & $P_{\text {mix }}=30.3 \mathrm{~mW}$ \\
$P_{\mathrm{IFA}}=20 \mathrm{~mW}$ & $P_{\text {syn }}=50 \mathrm{~mW}$ \\
$P_{\text {filt }}=P_{\text {filr }}=2.5 \mathrm{~mW}$ & $P_{\mathrm{LNA}}=20 \mathrm{~mW}$ \\
$N_{f}=10 \mathrm{~dB}$ & $M_{l}=40 \mathrm{~dB}$ \\
\hline
\end{tabular}

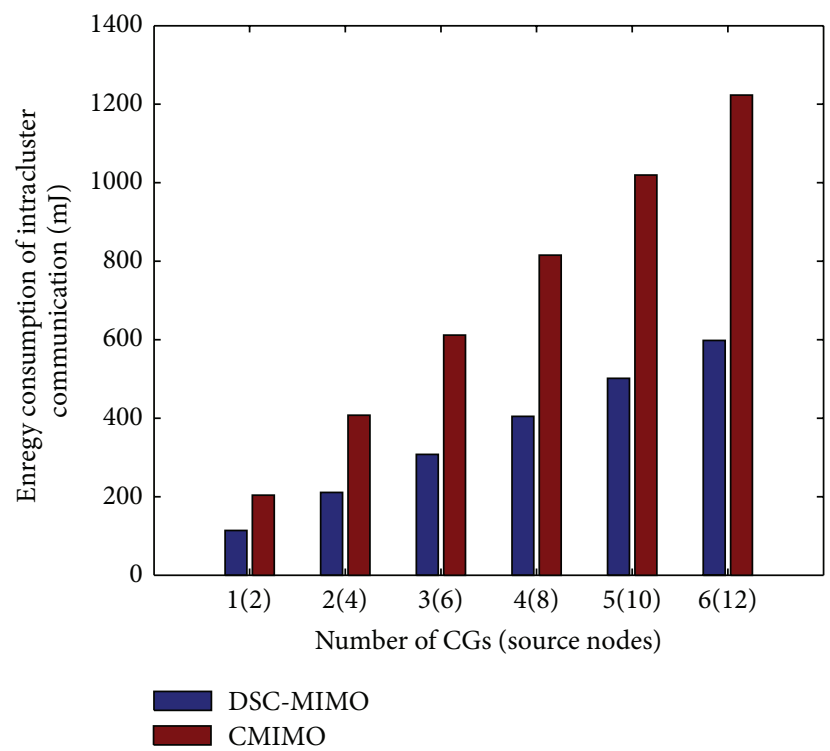

FIGURE 5: The energy consumption of DSC-MIMO and CMIMO in intracluster communication phase.

that DSC-MIMO consumes less energy than CMIMO when transmitting the same source data in intracluster communication phase. The gap between DSC-MIMO and CMIMO is growing with the increasing of source nodes. There are two reasons causing this increasing gap. One is that DSCMIMO improves the intracluster communication process and makes the source data forwarded only once, while CMIMO has to forward source data twice. Although DSC-MIMO increases some control overhead, the energy consumption of source data transmission is still the main part in this phase. The other is that the DSC is used in DSC-MIMO which can compress the related source data and reduce the transmission data. Therefore, DSC-MIMO is more energysaving than CMIMO in intracluster communication, and the performance is growing with the increasing number of CGs (source nodes).

Figure 6 shows the communication energy consumption of DSC-MIMO and CMIMO in the intercluster communication phase which reflects the energy consumption of one-hop virtual MIMO transmission. The result shows that DSC-MIMO consumes less energy than CMIMO when transmitting the same source data in intercluster communication phase. The gap between DSC-MIMO and CMIMO is growing with the increasing of source nodes which is not as big as intracluster communication. This is because the

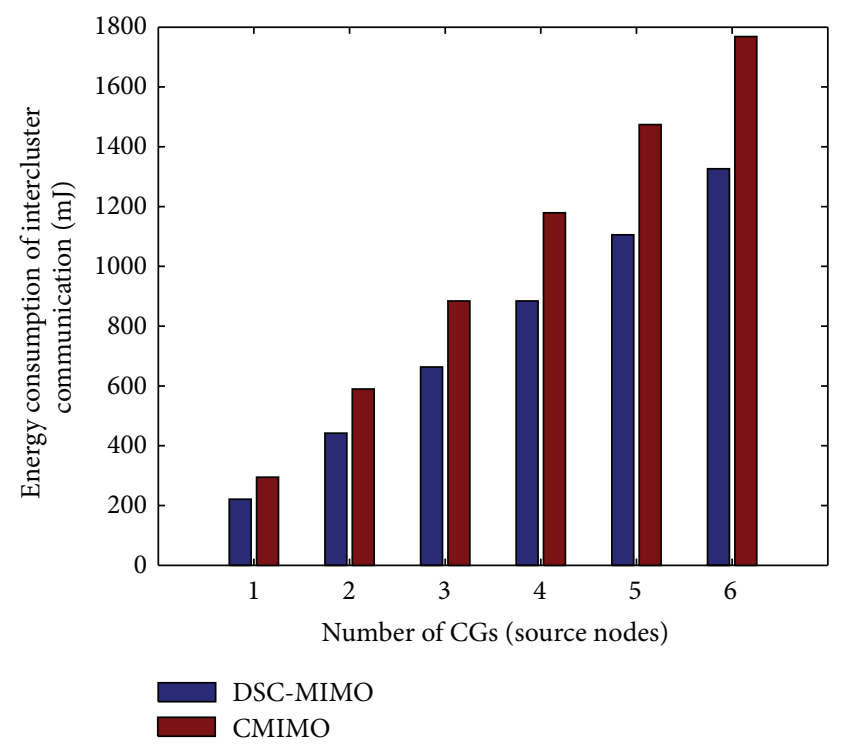

FIGURE 6: The energy consumption of DSC-MIMO and CMIMO in intercluster communication phase.

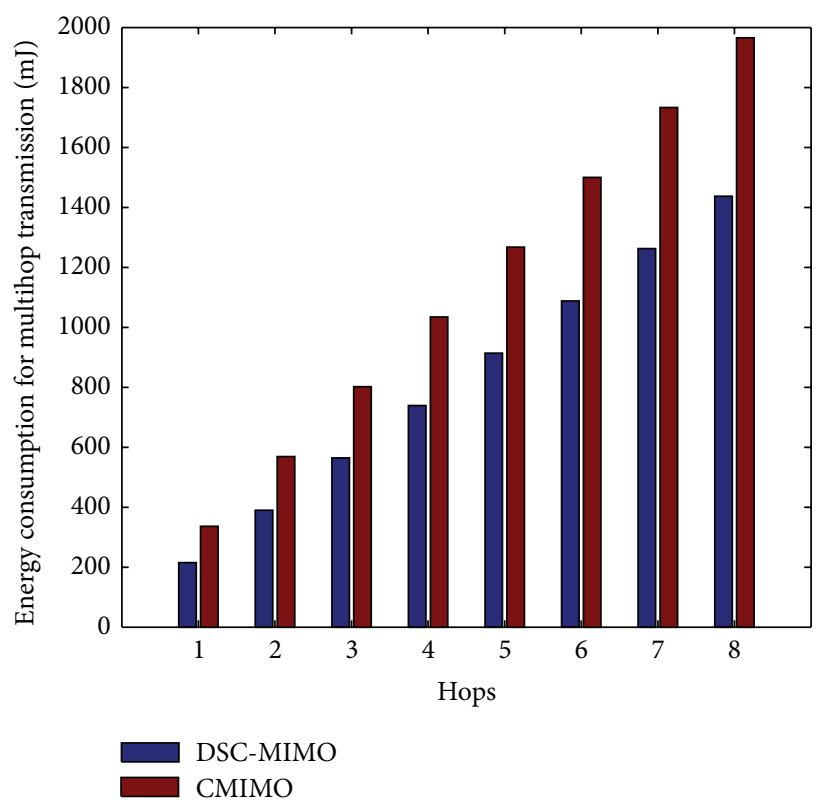

FIGURE 7: The energy consumption of DSC-MIMO and CMIMO in multihop transmission.

intercluster communication phase of DSC-MIMO is similar to CMIMO. However, the MCGs transmit the compressed data but do not decompress the data so it can reduce the energy consumption. Therefore, DSC-MIMO is more energysaving than CMIMO in intercluster communication, and the performance is growing with the increasing number of CGs (source nodes).

Figure 7 shows the communication energy consumption of DSC-MIMO and CMIMO in multihop virtual MIMO communication. During the simulation, we observe communication energy consumption of one CG (two source nodes) 


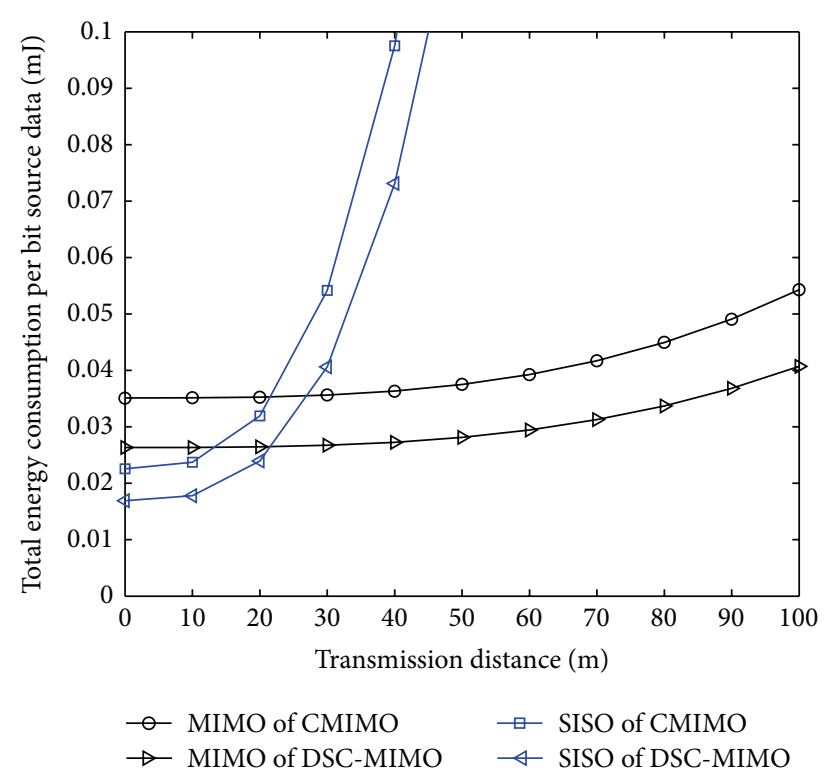

Figure 8: The energy efficiency of DSC-MIMO and CMIMO in different transmission modes.

each time and then calculate the average energy consumption after the multirepeated observation. The simulation result shows that energy consumption of DSC-MIMO is less than CMIMO. The gap is growing with the increasing number of hops because of the importing of DSC. This energy consumption gap is produced in both intracluster communication and intercluster communication. Therefore, DSC-MIMO is more energy-saving in multihop virtual MIMO communication than CMIMO.

Figure 8 shows the energy efficiency of DSC-MIMO and CMIMO in different transmission modes. We define the energy efficiency as the total energy consumption for transmitting 1-bit original source data. SISO is the transmission mode of intracluster communication and MIMO is the transmission mode of intercluster communication. The simulation result shows that the SISO of DSC-MIMO is more efficient than CMIMO and the MIMO of DSC-MIMO is also more efficient than CMIMO. It is mainly because of the DSC in DSC-MIMO which compresses the related source data and reduces the length of transmission data. Therefore, the DSC can improve the energy efficiency of high node density WSN.

\section{Conclusion}

Based on the existing virtual MIMO strategies for wireless sensor networks, we propose DSC-MIMO, which is very suitable for WSN with high node density and high scale, by improving intracluster communication and importing the DSC. Different to the existing virtual MIMO communication strategies, DSC-MIMO uses cooperative groups constituted by source nodes to transmit the data directly and the process of source data collecting and forwarding by cluster heads is removed, which can reduce the forwarding times of source data and energy consumption. The DSC can compress the related source data collected by adjacent nodes and improve the energy efficiency. The MCGs in DSC-MIMO can help WSN to realize the multihop virtual MIMO transmission by receiving and forwarding the data from CGs of other clusters. CG is the most basic communication unit in DSC-MIMO, so how to divide CGs has a great impact on the effectiveness of communication in WSN. The cooperative group allocation algorithm proposed in this paper can easily select the best allocation scheme enabling WSN to achieve the best communication performance.

Finally, by comparing the energy efficiency of DSCMIMO with CMIMO in simulation experiment, we prove that DSC-MIMO is more energy-saving and has higher energy efficiency.

\section{Conflict of Interests}

The authors declare that there is no conflict of interests regarding the publication of this paper.

\section{Acknowledgments}

This research has been supported by the Foundation of He'nan Educational Committee (nos. 14B120008 and 14A510003) and the National Natural Science Foundation of China (no. 61205090).

\section{References}

[1] N. Medhi and N. Sarma, "Mobility aided cooperative MIMO transmission in wireless sensor networks," Procedia Technology, vol. 6, pp. 362-370, 2012.

[2] S. Cui, A. J. Goldsmith, and A. Bahai, "Energy-efficiency of MIMO and cooperative MIMO techniques in sensor networks," IEEE Journal on Selected Areas in Communications, vol. 22, no. 6, pp. 1089-1098, 2004.

[3] S. K. Jayaweera, "Virtual MIMO-based cooperative communication for energy-constrained wireless sensor networks," IEEE Transactions on Wireless Communications, vol. 5, no. 5, pp. 984989, 2006.

[4] S. K. Jayaweera, "V-BLAST-based virtual MIMO for distributed wireless sensor networks," IEEE Transactions on Communications, vol. 55, no. 10, pp. 1867-1872, 2007.

[5] G. Bravos and A. G. Kanatas, "Energy efficiency comparison of MIMO-based and multihop sensor networks," EURASIP Journal on Wireless Communications and Networking, vol. 2008, Article ID 732145, 2008.

[6] Z. Rafique, B.-C. Seet, and A. Al-Anbuky, "Performance analysis of cooperative virtual MIMO systems for wireless sensor networks," Sensors, vol. 13, no. 6, pp. 7033-7052, 2013.

[7] M. Krunz, M. Z. Siam, and D. N. Nguyen, "Clustering and power management for virtual MIMO communications in wireless sensor networks," Ad Hoc Networks, vol. 11, no. 5, pp. 1571-1587, 2013.

[8] M. Nasim, S. Qaisar, and S. Lee, "An energy efficient cooperative hierarchical MIMO clustering scheme for wireless sensor networks," Sensors, vol. 12, no. 1, pp. 92-114, 2012.

[9] D. Slepian and J. K. Wolf, "Noiseless coding of correlated information sources," IEEE Transactions on Information Theory, vol. 19, pp. 471-480, 1973. 
[10] S. S. Pradhan and K. Ramchandran, "Distributed source coding: symmetric rates and applications to sensor networks," in Proceedings of the IEEE Data Compression Conference, pp. 363-372, March 2000.

[11] J. Garcia-Frias and Y. Zhao, "Compression of correlated binary sources using turbo codes," IEEE Communications Letters, vol. 5, no. 10, pp. 417-419, 2002.

[12] A. D. Liveris, Z. Xiong, and C. N. Georghiades, "A distributed source coding technique for correlated images using turbocodes," IEEE Communications Letters, vol. 6, no. 9, pp. 379-381, 2002.

[13] A. D. Liveris, Z. Xiong, and C. N. Georghiades, "Compression of binary sources with side information at the decoder using LDPC codes," IEEE Communications Letters, vol. 6, no. 10, pp. 440-442, 2002.

[14] D. Schonberg, K. Ramchandran, and S. S. Pradhan, "Distributed code constructions for the entire Slepian-Wolf rate region for arbitrarily correlated sources," in Proceedings of the IEEE Data Compression Conference (DCC '04), pp. 292-301, IEEE, March 2004.

[15] M. Sartipi and F. Fekri, "Distributed source coding in wireless sensor networks using LDPC coding: the entire Slepian-Wolf rate region," in Proceedings of the Wireless Communications and Networking Conference, vol. 4, pp. 1939-1944, IEEE, March 2005.

[16] M. Sartipi and F. Fekri, "Distributed source coding using short to moderate length rate-compatible LDPC codes: the entire Slepian-Wolf rate region," IEEE Transactions on Communications, vol. 56, no. 3, pp. 400-411, 2008.

[17] Q. Qu, L. B. Milstein, and D. R. Vaman, "Cooperative and constrained MIMO communications in wireless ad hoc/sensor networks," IEEE Transactions on Wireless Communications, vol. 9, no. 10 , pp. 3120-3129, 2010. 

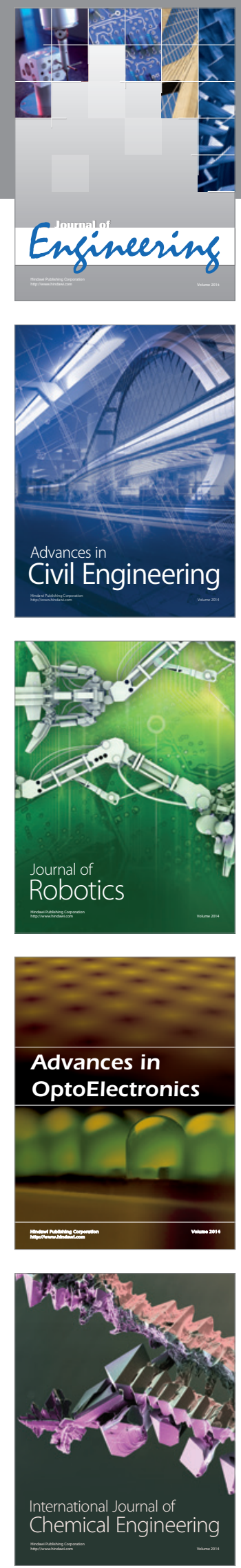

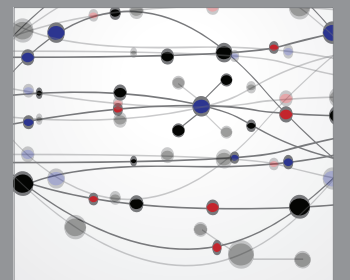

The Scientific World Journal
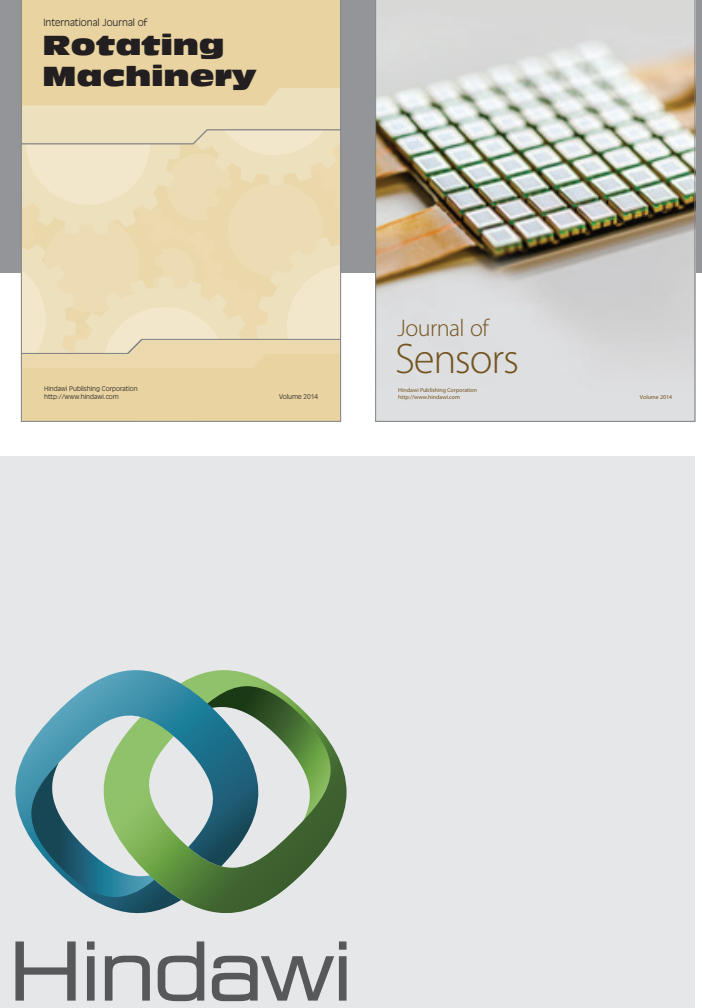

Submit your manuscripts at http://www.hindawi.com
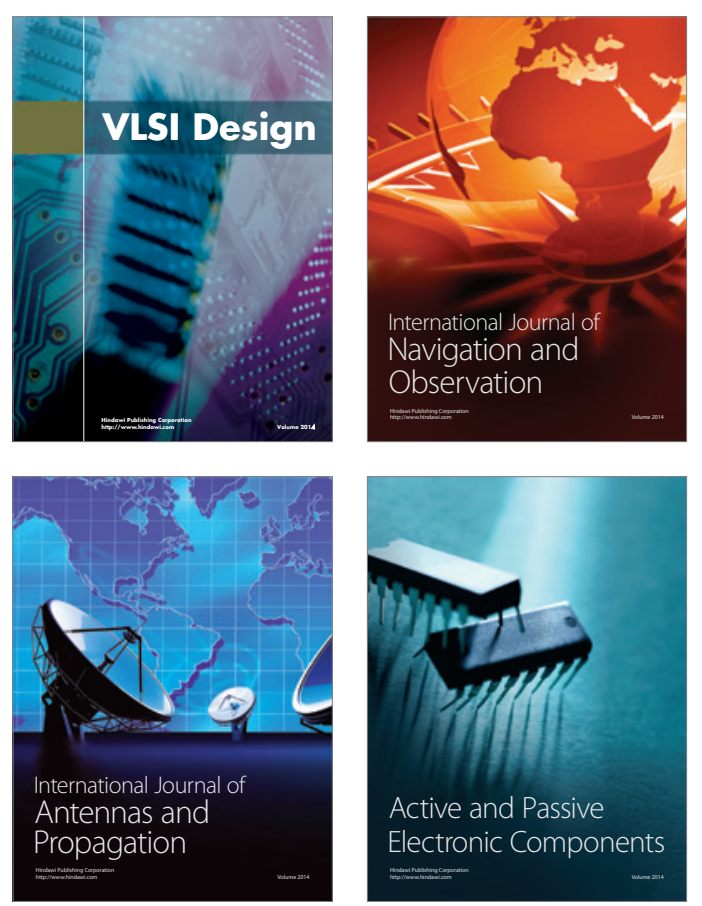
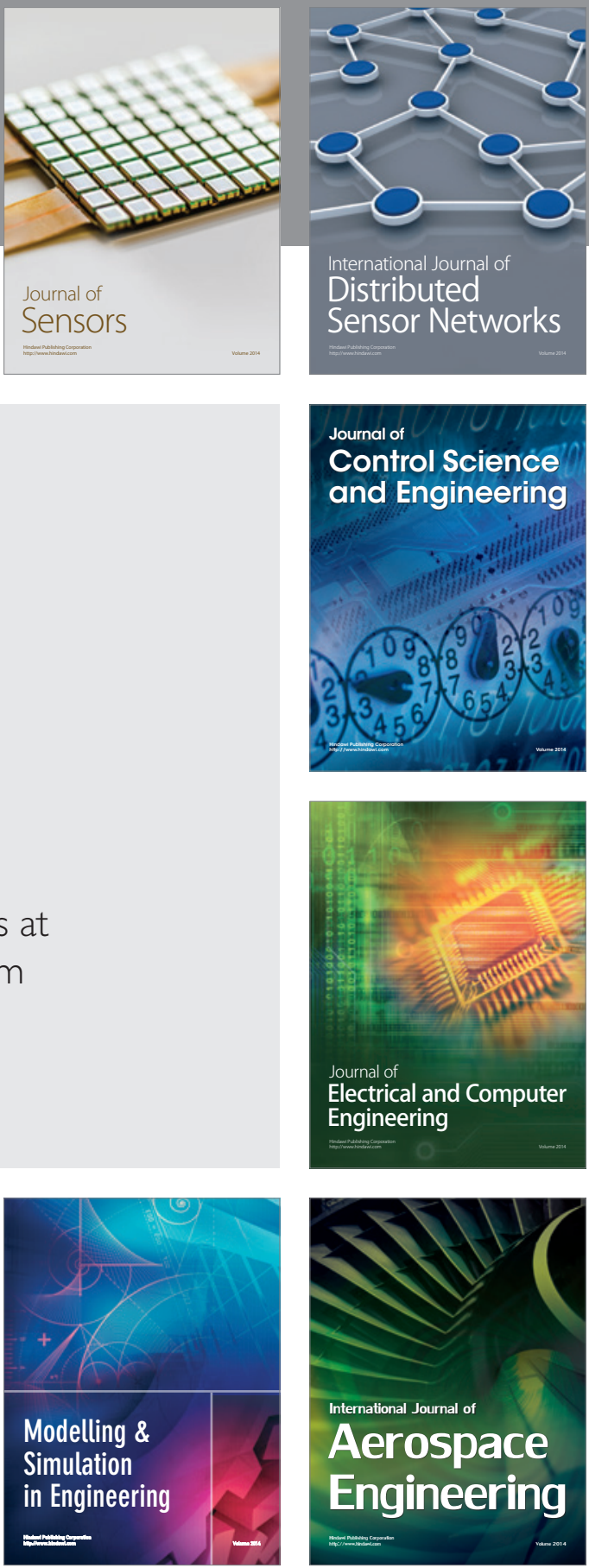

Journal of

Control Science

and Engineering
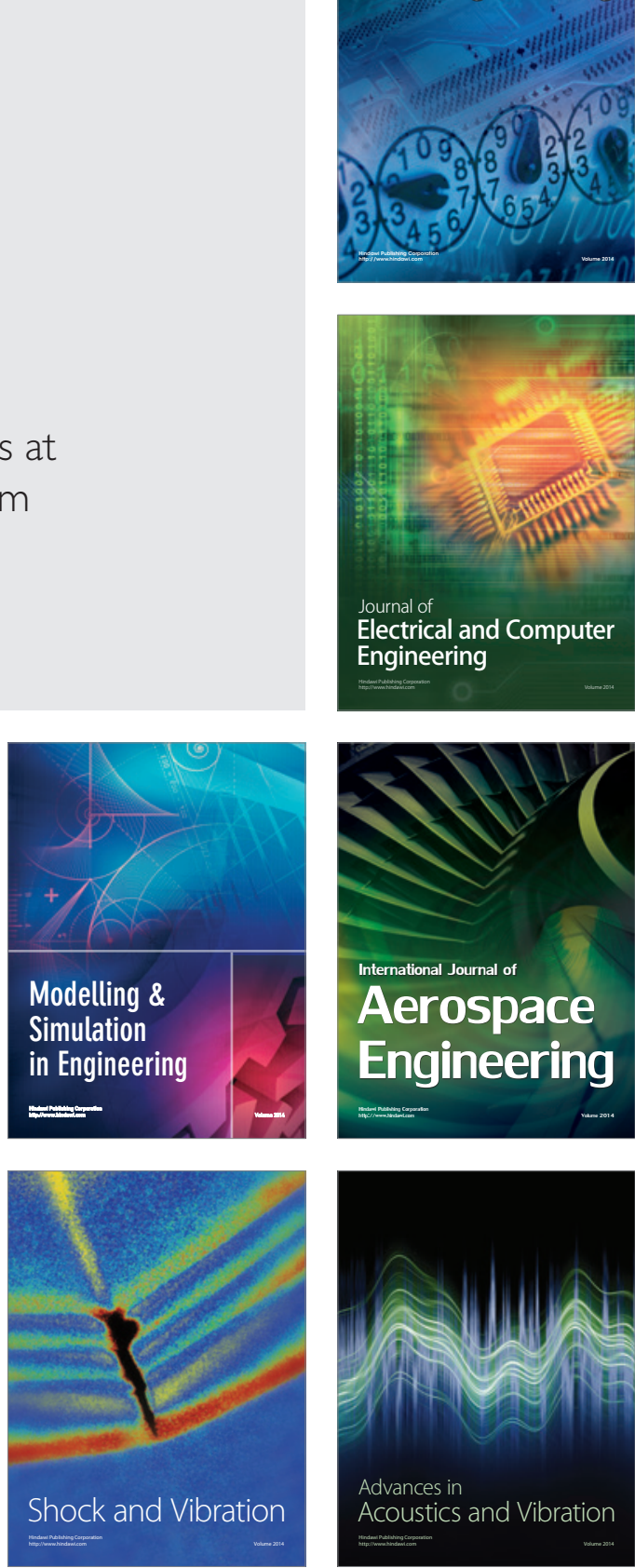